Research Paper

\title{
Systemic neutrophil lymphocyte ratio and mismatch repair status in colorectal cancer patients: correlation and prognostic value
}

\author{
Wen-Zhuo He1\#, Wan-Ming Hu2,3,4\#, Peng-Fei Kong1\#, Lin Yang1, Yuan-Zhong Yang2, Qian-Kun Xie1,

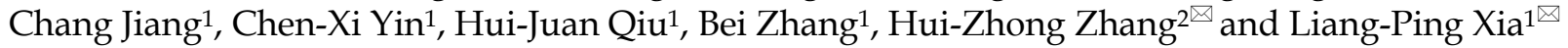 \\ 1. VIP Region, Sun Yat-sen University Cancer Center; State Key Laboratory of Oncology in South China; Collaborative Innovation Center for Cancer Medicine, \\ Guangzhou, Guangdong 510060, P. R. China \\ 2. Department of Pathology,SunYat-sen University Cancer Center; State Key Laboratory of Oncology in South China; Collaborative Innovation Center for \\ Cancer Medicine, Guangzhou, Guangdong 510060, P. R. China \\ 3. Department of Pathology, School of Basic Medical Sciences, Southern Medical University, Guangzhou 510515, China \\ 4. Department of Pathology, Nanfang Hospital, Southern Medical University, Guangzhou 510515, China \\ \#Wen-Zhuo He, Wan-Ming Hu and Peng-Fei Kong contributed equally to this paper.
}

$\triangle$ Corresponding authors: Dr. Liang-Ping Xia, Email: xialiangping@163.com; Fax: +8620-87343392; Tel: +8620-87343107; Mobile: 13926410608. Addresses: Sun Yat-sen University Cancer Center; State Key Laboratory of Oncology in South China; Collaborative Innovation Center for Cancer Medicine, 651 Dongfeng East Road, Guangzhou, Guangdong 510060, P. R. China and Dr. Hui-Zhong Zhang, Email: zhanghuizh@sysucc.org.cn; Fax: +8620-87343392; Tel: +8620-87342701. Addresses: Sun Yat-sen University Cancer Center; State Key Laboratory of Oncology in South China; Collaborative Innovation Center for Cancer Medicine, 651 Dongfeng East Road, Guangzhou, Guangdong 510060, P. R. China.

(c) Ivyspring International Publisher. This is an open access article distributed under the terms of the Creative Commons Attribution (CC BY-NC) license (https://creativecommons.org/licenses/by-nc/4.0/). See http://ivyspring.com/terms for full terms and conditions.

Received: 2018.04.13; Accepted: 2018.07.04; Published: 2018.08.06

\begin{abstract}
Purpose: Mismatch repair-deficient (dMMR) colorectal cancer (CRC) is associated with increased local immune response as compared with mismatch repair-proficient (PMMR) CRC. We evaluated the relationship between MMR status and systemic inflammatory factors, including neutrophil lymphocyte ratio (NLR) and C-reactive protein (CRP). We also assessed the prognostic value of these parameters.

Methods and materials: We analysed the relationship between MMR status (obtained by histochemical analysis), neutrophil and lymphocyte counts, NLR, and CRP level. The impact of systemic inflammatory factors on survival was also evaluated in DMMR and PMMR CRC patients.

Results: A total of 1353 male and 892 female patients were eligible for analysis, of which, 253 patients $(11.3 \%)$ were found to have dMMR status. Patients with dMMR status presented with increased neutrophil counts, and higher NLR and CRP levels in early stage CRC. In stage IV CRC patients, no correlation between MMR status and systemic inflammatory factors was found. Lymphocyte counts did not correlate with MMR status. High NLR was a prognostic factor for poor survival in PMMR CRC. However, NLR was not a prognostic factor in AMMR CRC.

Conclusions: Our results suggest that $\mathrm{dMMR} C R C$ correlates with higher neutrophil count, NLR and CRP levels only in non-metastatic patients, and NLR has prognostic value only in PMMR CRC.
\end{abstract}

Key words: NLR; CEA; colorectal cancer; biomarker; prognosis

\section{Introduction}

Recent studies have highlighted the role of mismatch repair (MMR) status as a prognostic factor for survival and a predictor for PD-1 blockade in metastatic colorectal cancer (CRC) [1-3]. Tumours with MMR-deficiency (dMMR) are known to display several differentiating characteristics, such as mucinous features, and presence of high levels of tumour-infiltrating lymphocytes [4-7]. Indeed, several studies have reported differences in immune microenvironment and genetic characteristics 
between dMMR and MMR-proficient (pMMR) tumours [8, 9]. For example, Llosa et al. report increased mutational burden as well as T-helper cell 1 (Th1) and cytotoxic T-lymphocyte (CTL) infiltration in dMMR tumours [9].

Apart from local immune response, there is growing consensus that systemic inflammatory factors also promote cancer growth and metastasis [10-15]. For example, increases in both neutrophils and C-reactive protein (CRP) were associated with shorter survival in metastatic colorectal cancer (mCRC patients) [10, 16]. Although there is an accepted link between MMR status and local inflammatory factors, it is unclear whether MMR status is associated with systemic inflammatory response, since conflicting evidence exists in literature. While Pine et al found no association between MMR status and neutrophil lymphocyte ratio, Park and group reported that both neutrophil counts and CRP levels were higher in dMMR patients $[12,17]$. The inconsistency in results might be caused by small sample size and heterogeneity in tumour stages of the patients.

In light of these aspects, we conducted a study aimed at overcoming the abovementioned limitations and set out to investigate the relationship between MMR status and systemic inflammatory factors in CRC patients. In addition, we also conducted subgroup analysis based on tumour stage.

\section{Materials and methods}

\section{Patient selection}

We selected patients who met the following inclusion criteria: 1) diagnosis of colorectal cancer at the Sun Yat-sen University Cancer Center between 2009 and 2014; 2) availability of pre-treatment routine blood test records; and 3) availability of follow-up information; 4) underwent resection, including both curative and palliative intent. The exclusion criteria included presence of infection, haematological disease, hyperpyrexia, intestinal perforation, or intestinal obstruction at initial diagnosis. Patients with emergency resection or managed non-operatively were excluded. Informed consent was obtained from all patients. This study was approved by the ethical committee of Sun Yat-sen University cancer center.

\section{Measurement of neutrophil counts, lymphocyte counts, and CRP and carcino-embryonic antigen (CEA) levels}

Neutrophil and lymphocyte counts were part of routine blood test parameters. The routine blood tests were conducted by SysmexXE-5000 ${ }^{\mathrm{TM}}$ Automated
Hematology System (Shanghai, China), while CRP quantitation was performed using a Hitachi Automatic Analyzer 7600-020 (Tokyo, Japan). A Roche Elecsys 2010 Chemistry Analyzer (Basel, Switzerland) was utilised for the electrochemiluminescent measurement of carcinoembryonic antigen (CEA) levels. A ratio of the neutrophil counts to the lymphocyte counts, defined as the neutrophil to lymphocyte ratio (NLR) was calculated, and the samples were categorized into two groups based on a NLR ratio of $>3$ and that of $\leq 3$.

\section{Assessment of MMR status}

The slides were obtained from the archives of the Department of Pathology, Cancer Center, Sun Yat-Sen University, Guaangzhou, China. The tumour cases were histologically re-confirmed and we selected the most representative block for immunohistochemistry (IHC). IHC for the four most commonly assessed mismatch repair proteins(MutL homolog 1 [MLH1], postmeiotic segregation increased, Saccharomycescerevisiae 2 [PMS2],MutS homolog 2 [MSH2], and MutS homolog 6 [MSH6])was performed using the Envision two-step procedure, as per manufacturer's instructions (Dako, Glostrup, Denmark). The tumour tissue sections were dried overnight at $37^{\circ} \mathrm{C}$, dewaxed in xylene, rehydrated by passing through graded alcohol, and immersed in 3\% hydrogen peroxide for $20 \mathrm{~min}$ to block endogenous peroxidase activities. The tissue sections were then pre-treated with antigen retrieval buffer (EDTA buffer, $\mathrm{pH} \mathrm{8.0,} \mathrm{at} 100^{\circ} \mathrm{C}, 2 \mathrm{~min}$ in a pressure cooker), and incubated with $10 \%$ normal goat serum at room temperature for $10 \mathrm{~min}$ to block nonspecific signals. Subsequently, the slides were incubated overnight at $4^{\circ} \mathrm{C}$ using the following primary antibodies: anti-MLH1, anti-PMS2, anti-MSH2, and anti-MSH6 (1:50; Beijing Zhong Shan-Golden Bridge Biological Technology, Beijing, China). After rinsing five times with $0.01 \mathrm{~mol} / \mathrm{L}$ phosphate-buffered saline (PBS; $\mathrm{pH}=7.4$ ) for $10 \mathrm{~min}$, the sections were incubated with a secondary antibody (Envision; Dako, Glostrup, Denmark) for 1 hour at $25^{\circ} \mathrm{C}$, washed again in PBS, and stained with DAB (3,3-diaminobenzidine) substrate. Finally, the sections were counterstained with Mayer's haematoxylin, dehydrated, and mounted. The negative control samples were treated with PBS instead of the primary antibody. Non-neoplastic colonic mucosa, stromal cells, infiltrating lymphocytes, or the centres of lymphoid follicles were used as internal positive controls. Known MMR deficient colorectal carcinomas served as external negative controls. Two experienced pathologists blinded to patient clinical profile evaluated the staining results independently. Positive protein 
expression was defined as nuclear staining within tumour cells, while negative protein expression was defined as complete absence of nuclear staining with concurrent positive signal from internal positive control staining. Tumours with loss of MLH1, PMS2, MSH2, or MSH6 protein, as visualized by light microscopy, were classified as MLH1, PMS2, MSH2, or MSH6 negative, respectively. If internal non-neoplastic tissues showed invalid negative staining, the IHC procedure was repeated. When the opinions of the two pathologists were different, agreement was reached by consensus.

\section{Assessment of local tumour microenvironment}

The tumour microenvironment was evaluated by histopathological analysis of haematoxylin and eosin (HE) stained tissue sections by two pathologists who were blinded to the clinical data. In case of differences of opinion, agreement was reached by consensus. We evaluated all the HE sections for each patient. In general, the inflammatory responses in different sections of a tumour were similar. If there were differences between sections, the most representative and invasive part of the primary tumour was chosen in order to avoid bias. Tumour microenvironment parameters were assessed as previously reported [17-19]. We studied the stromal percentage, (SP, defined as the stromal-carcinoma ratio as visible in the tumour section), neutrophil and lymphocyte infiltration (both in central region and invasive margin), and Crohn's-like inflammatory reaction. Briefly, SP 70\% implies a stromal percentage of $70 \%$; SP was classified as SP-low $(<50 \%)$ and SP-high $(\geq 50 \%)$. We used a four-degree scale to assess the infiltration of neutrophils and lymphocytes. A score of 0 indicated an absence of reaction, 1 weak, 2 moderate, and 3 severe increase of each cell type. Absent and weak (0-1) were combined as low grade, while moderate to severe increase (2-3) were high grade. Crohn's-like reaction was defined as transmural lymphoid reaction, often involving the lymphoid follicle-like structures. It was scored as 0 (absent) and 1(existent).

\section{Patient follow-up and statistical analysis}

Disease-free survival (DFS), defined as the time from diagnosis to the time of the first event (loco regional recurrence, metastasis, or death), and overall survival (OS), defined as the time from diagnosis to the date of death or the last date of follow-up, were evaluated in this study. The last follow-update was 31 August, 2016.

All statistical analyses were performed using the Statistical Package for the Social Sciences (SPSS) software, version 22. Frequency distribution analysis and descriptive statistics were used to compare patient characteristics. Survival curves were plotted using the Kaplan-Meier method, and the differences were compared using the loge-rank test. Multivariate analysis using a Cox proportional hazards model was used to test independent significance by backward elimination of insignificant explanatory variables. A P value $\leq 0.05$ was considered to be significant.

\section{Results}

\section{Patient characteristics}

The details of clinic-pathological features of patients in the study are shown in Table 1. The study included 1353 male patients and 892 female patients, with a median age of 58 (ranging from 18 years to 87 years). Among the cases included, there were $572(25.5 \%)$ right-sided colon cancers, $873(38.9 \%)$ left-sided colon cancers, and 775 (34.5\%) rectal cancers. As shown in Table 1, there were 304(13.5\%) stage I, 874 (38.9\%) stage II, $543(24.2 \%)$ stage III, and $524(23.3 \%)$ stage IV patients. As classified by MMR status, $253(11.3 \%)$ were identified as dMMR, while $1992(88.7 \%)$ were pMMR.

Table 1: Patient characteristics

\begin{tabular}{|c|c|c|c|c|}
\hline Variable & Total & MMR-deficient & MMR-proficient & $p$ \\
\hline Number of patients & 2245 & 253 & 1992 & \\
\hline \multicolumn{5}{|l|}{ Age } \\
\hline$\leq 50 \mathrm{y}$ & 669 & 108 & 561 & $<0.001^{*}$ \\
\hline $51-65 y$ & 987 & 97 & 890 & \\
\hline$>65 y$ & 589 & 48 & 541 & \\
\hline \multicolumn{5}{|l|}{ Sex } \\
\hline Male & 1353 & 157 & 1196 & 0.585 \\
\hline Female & 892 & 96 & 796 & \\
\hline \multicolumn{5}{|l|}{ Mucinous histology } \\
\hline Yes & 272 & 59 & 213 & $<0.001^{*}$ \\
\hline No & 1973 & 194 & 1779 & \\
\hline \multicolumn{5}{|l|}{ Stage } \\
\hline I & 304 & 32 & 272 & $0.004^{*}$ \\
\hline II & 874 & 118 & 756 & \\
\hline III & 543 & 40 & 503 & \\
\hline IV & 524 & 63 & 461 & \\
\hline \multicolumn{5}{|c|}{ Primary tumor location } \\
\hline Right-side colon & 572 & 122 & 450 & $<0.001^{*}$ \\
\hline Left-side colon & 873 & 66 & 807 & \\
\hline Rectum & 775 & 54 & 721 & \\
\hline Multiple & 25 & 11 & 14 & \\
\hline \multicolumn{5}{|l|}{ CEA } \\
\hline$\leq 5 \mathrm{ng} / \mathrm{ml}$ & 1253 & 151 & 1102 & 0.092 \\
\hline$>5 \mathrm{ng} / \mathrm{ml}$ & 975 & 98 & 877 & \\
\hline unknown & 17 & 4 & 13 & \\
\hline
\end{tabular}

* Chi-square test was used to compare patient characteristics, and a P value $\leq 0.05$ was considered to be significant.

\section{MMR status and systemic inflammatory factors}

The correlation between MMR status and systemic inflammatory factors is shown in Table 2. We evaluated stage I and II disease patients as a subgroup, since the number of stage I patients was 
relatively small. Both NLR and CRP parameters were more likely to be elevated in dMMR patients. However, subgroup analysis revealed that the correlation between MMR status and systemic inflammatory factors was dependent on tumour stage. The relationship sustained from stage I to III, but was not statistically significant in stage IV patients, as shown in Table 2.

Table 2: Relationship between MMR status and systemic inflammatory response

\begin{tabular}{|c|c|c|c|}
\hline & No. of MMR-deficient & No. of MMR-proficient & $P$-value, chi-square \\
\hline \multicolumn{4}{|c|}{ All patients } \\
\hline NLR & & & $0.007^{*}$ \\
\hline$\leq 3$ & 154 & 1370 & \\
\hline$>3$ & 99 & 622 & \\
\hline CRP मे & & & $<0.001^{*}$ \\
\hline normal & 144 & 1442 & \\
\hline elevated & 105 & 538 & \\
\hline \multicolumn{4}{|l|}{ Stage I + II } \\
\hline NLR & & & $0.044^{*}$ \\
\hline$\leq 3$ & 93 & 713 & \\
\hline$>3$ & 57 & 315 & \\
\hline CRP & & & $<0.001^{*}$ \\
\hline normal & 83 & 765 & \\
\hline elevated & 67 & 259 & \\
\hline \multicolumn{4}{|l|}{ Stage III } \\
\hline NLR & & & $0.033^{*}$ \\
\hline
\end{tabular}

\begin{tabular}{|c|c|c|c|}
\hline & No. of MMR-deficient & No. of MMR-proficient & $P$-value, chi-square \\
\hline$\leq 3$ & 24 & 377 & \\
\hline$>3$ & 16 & 126 & \\
\hline CRP & & & $0.004^{*}$ \\
\hline normal & 24 & 404 & \\
\hline elevated & 16 & 99 & \\
\hline Stage IV & & & \\
\hline NLR & & & 0.430 \\
\hline$\leq 3$ & 37 & 280 & \\
\hline$>3$ & 26 & 181 & \\
\hline CRP & & & 0.416 \\
\hline normal & 37 & 273 & \\
\hline elevated & 22 & 180 & \\
\hline
\end{tabular}

${ }^{*}$ Chi-square test was used to compare patient characteristics, and a P value $\leq 0.05$ was considered to be significant.

is 16 patients were with missing CRP values.

Further, we compared the distribution of neutrophil and lymphocyte counts, and CRP levels between $\mathrm{dMMR}$ and $\mathrm{pMMR}$ patients, as grouped by tumour stage (Figure 1). Patients with dMMR status had higher neutrophil counts and CRP levels as than those with pMMR status, when distant metastasis was absent. In stage IV patients, neutrophil and CRP levels did not correlate with MMR status. The distribution of lymphocyte counts was similar in all groups of patients.
A

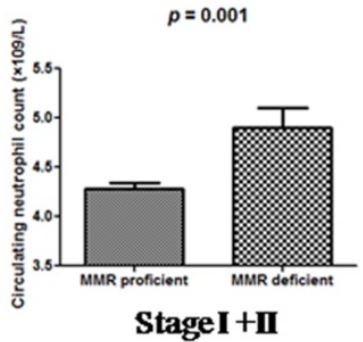

D
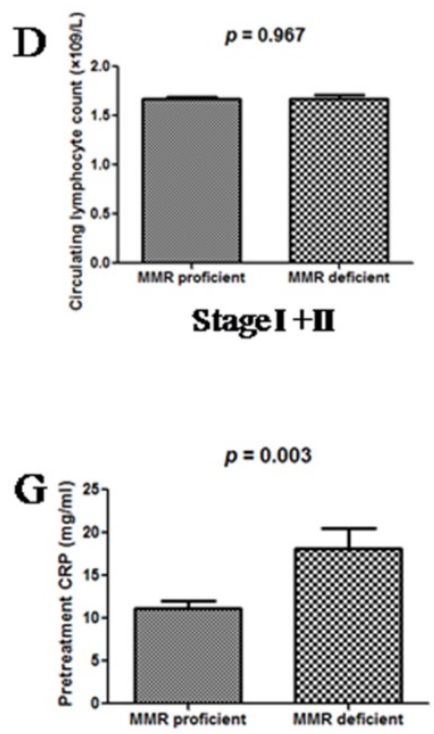

Stage I +II
B

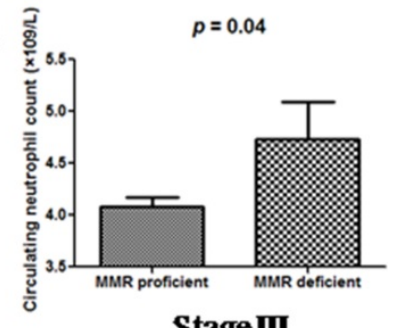

Stage III

E

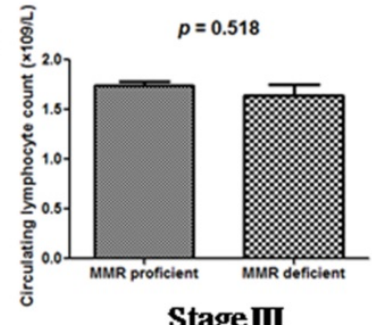

Stage III

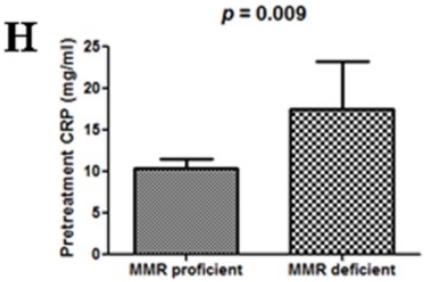

Stage III

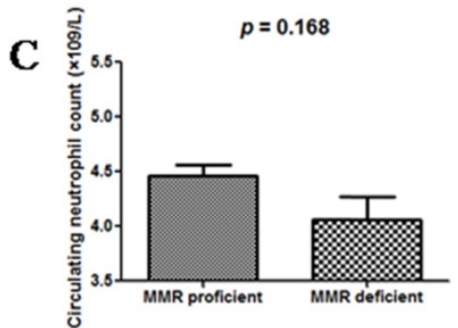

StageIV

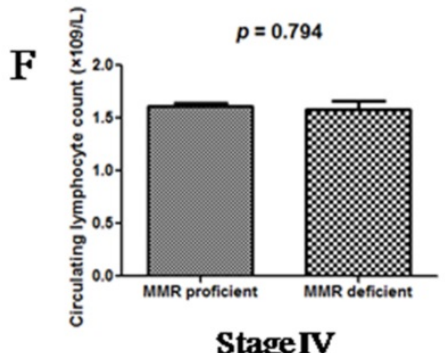

StageIV

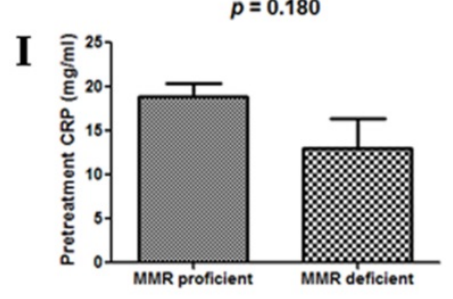

StageIV

Figure 1: The distributions of neutrophil counts (A, B and C), lymphocyte counts (D, E and F) and CRP levels ( $G, H$ and I) between dMMR and pMMR CRC patients, as grouped by tumour stage. 
A

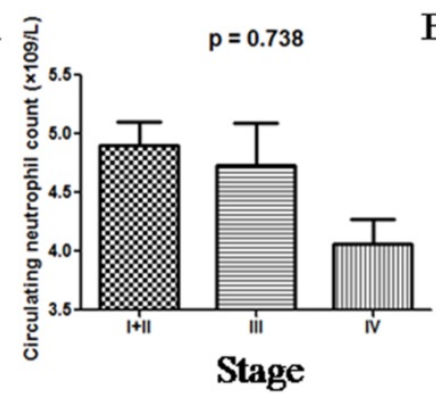

D

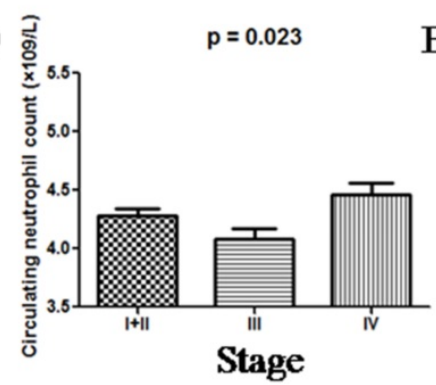

B
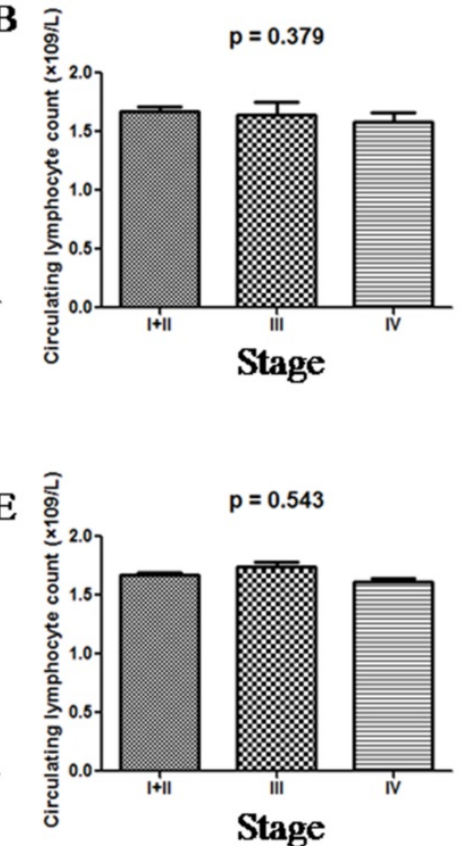

C
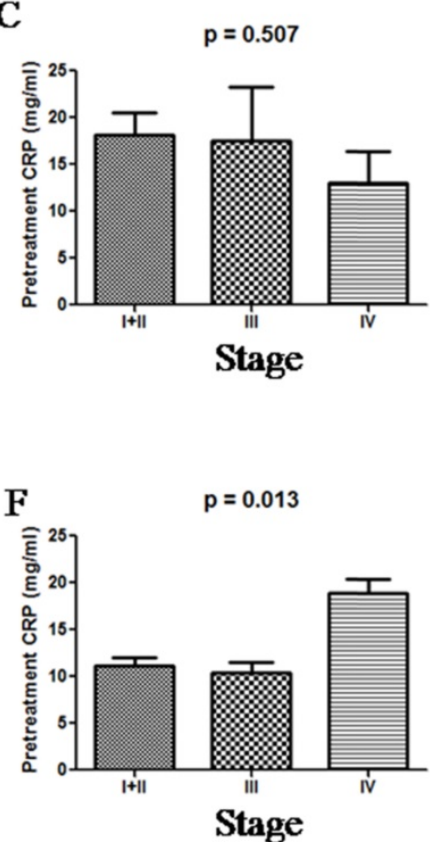

Figure 2: The distributions of neutrophil counts, lymphocyte counts and CRP in dMMR (A, B and C) and pMMR (D, E and F) CRC patients as grouped by stage.

Diabetes mellitus and hypertension are commonly found in colon cancer patients, and they influence the levels of CRP or neutrophil counts. We performed sub-group analysis in 1784 patients without hypertension and 2048 non-diabetic patients. The distribution of NLR and CRP levels between $\mathrm{dMMR}$ and pMMR patients was similar (data not shown).

When grouped by tumour stage, dMMR patients had similar neutrophil and lymphocyte counts, and CRP levels. Unlike dMMR patients, pMMR patients exhibited significantly higher neutrophil counts and CRP levels when distant metastasis was found, as shown in Figure 2. Lymphocyte counts were similar among all pMMR patients regardless of tumour stage.

\section{Systemic inflammatory factors and local tumour environment}

We sought to understand the relationship between local inflammation within the tumour microenvironment and systemic inflammation, while assessing the effect of MMR status on this relationship. Since most rectal cancer patients accepted radiotherapy and chemotherapy before surgery, we excluded rectal cancer patients when evaluating the primary tumour specimens. Among the 1445 colon cancer patients, 285 patients were excluded for non-availability of pathological specimens. Finally, 1160 colon cancer patients were included.

We first evaluated the correlation between local tumour environment and MMR status. As previously reported, dMMR tumours displayed significantly increased neutrophil infiltration, lymphocytes infiltration, and Crohn's-like lesions (Supplementary table 1). Additionally, colon cancer patients with increased serum CRP levels were more likely to have a lower stromal percentage, higher density of neutrophil infiltration, less lymphocyte infiltration, and a higher chance of Crohn's-like reaction (Supplementary table 2). A NLR $>3$ was a predicator of higher stromal percentage, and higher density of neutrophil infiltration.

We also analysed the effect of MMR status on the relationship between local inflammatory infiltrates in the primary tumour, and systemic inflammation. In dMMR patients, CRP levels correlated only with lymphocyte infiltration, within the tumour microenvironment parameter panel, while no correlation was observed between NLR and local tumour microenvironment parameters (Supplementary table 3). In pMMR patients, increased serum CRP levels were predictive of higher density of neutrophils infiltration, lower lymphocyte infiltration, and a higher chance of Crohn's-like reaction. A NLR $>3$ was predictive of higher neutrophil infiltration in the central region (Supplementary table 4).

\section{Survival grouped by systemic inflammatory factors and MMR status}

To understand the effect of systemic inflammatory factors and MMR status on survival parameters, we statistically analysed data from both $\mathrm{dMMR}$ and $\mathrm{pMMR}$ patients. We first focused on stage I + II CRC patients. As previously reported, NLR is inversely related to DFS. In patients with $N L R>3$, 
median DFS (34.42 months) was significantly lower than that in NLR $\leq 3$ population (35.53 months; (hazard ratio (HR):1.588, 95\%HR: 1.100-2.293; $p=$ 0.014). We further found that NLR was not significantly associated with DFS in dMMR patients (median DFS in the NLR $>3$ population was 40.40 versus 37.57 months in the NLR $\leq 3$ population, HR: 1.79; 95\%HR: $0.762-4.231 ; p=0.188$ ). The prognostic value of NLR sustained in pMMR patients (median DFS in the NLR>3 population was 33.63 versus 35.13 months in the NLR $\leq 3$ population, HR: $1.53 ; 95 \% \mathrm{HR}$ : 1.015-2.307 $p=0.023$ ). Similar results were also observed in stage III patients, as shown in Figure 3.

In stage IV patients, NLR was not a prognostic factor when MMR status was dMMR (median OS in the NLR $>3$ population was 37.4 versus 40.2 months in the NLR $\leq 3$ population, HR: $1.10 ; p=0.812$ ). While in pMMR patients, NLR was a predictive factor for patient survival (median OS in the NLR $>3$ population was 22.8 versus 27.4 months in the NLR $\leq 3$ population, HR: $1.33 ; p=0.032$ ). The survival curves are shown in Figure 4. Taken together, these results indicate that NLR is not a predictive parameter when the MMR status is dMMR. Since NLR had prognostic value in pMMR patients, we performed multivariate analysis only in the pMMR subgroup and found that the prognostic value of NLR was independent of age, gender, tumour stage and CEA level (HR: 1.48; 95\% confidence interval: 1.13 to $1.93 ; p=0.004)$.

\section{Discussion}

In this study, we sought to establish the relationship between MMR status, local tissue inflammatory microenvironment, and systemic inflammation, and the overall effect of these factors on patient survival parameters. Our results indicate that systemic inflammatory factors, including NLR and CRP, are associated with MMR status. Higher neutrophil counts and CRP levels were observed in dMMR CRC, than in PMMR CRC, though this was only in non-metastatic CRC. Both NLR and CRP were comparable between dMMR and pMMR CRC when metastases were found. In pMMR CRC patients, NLR and CRP had significant prognostic value. These results suggest that systemic inflammatory factors are affected by MMR status and tumour stage (related to distant metastasis).
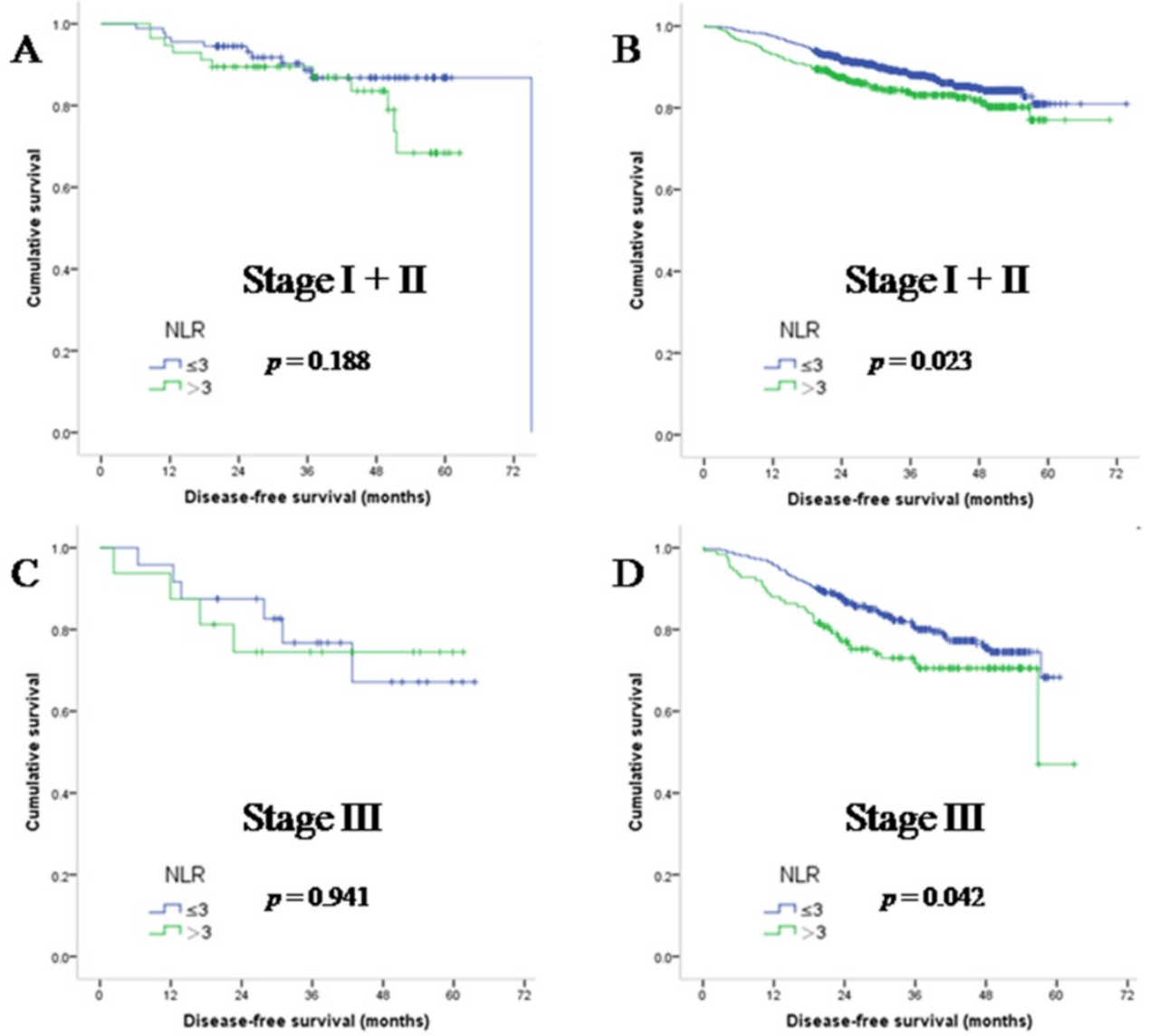

Figure 3: Disease-free survival of early stage CRC patients with dMMR (A and $C$ ) and PMMR (B and $D)$ status, as grouped by NLR. 

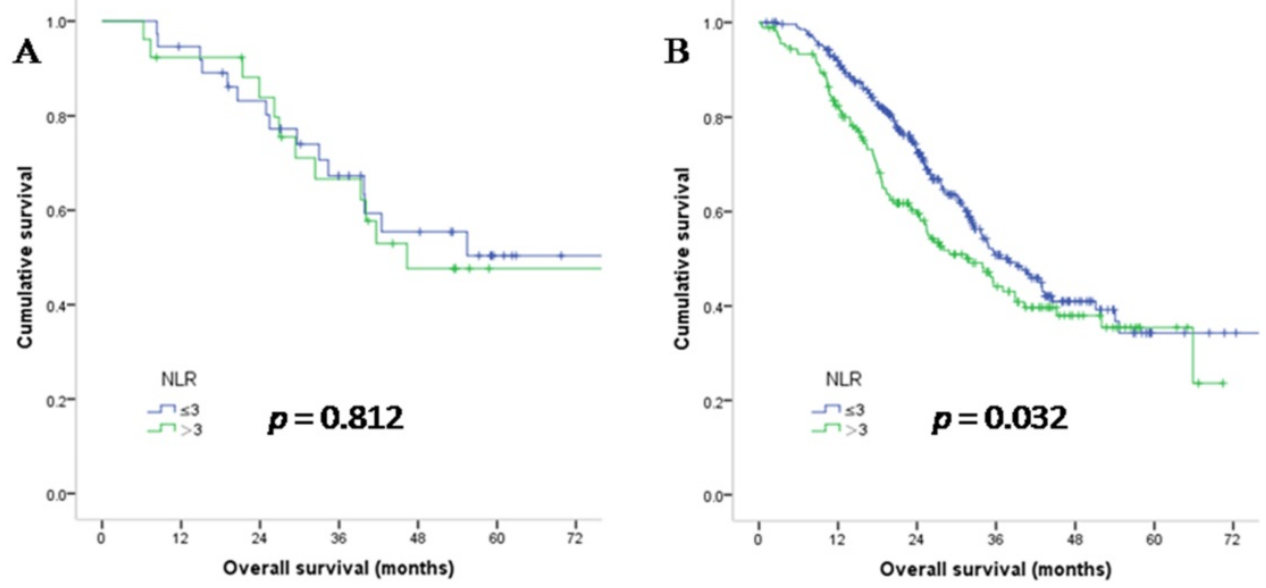

Figure 4: Overall survival of stage IV CRC patients with $\mathrm{DMMR}(\mathrm{A})$ and pMMR (B) status, as grouped by NLR.

The exact mechanism underlying the correlation between systemic inflammatory factors and MMR status is unclear, and several possible explanations can be derived from literature. Firstly, Llosa et al report that the tumour microenvironment in dMMR colon cancer is balanced by various counter-inhibitory checkpoints [9]. Casbon et al reported that soluble tumour-derived factors may reprogram myeloid differentiation and increase production of neutrophils, which preferentially accumulate in the tumour to promote a local immune suppressive environment [20]. Thus, increased neutrophil count in dMMR CRC patients might reflect an immunosuppressive microenvironment. Secondly, Missiaglia et al reported an association between MMR colon carcinomas and expression of genes related to inflammatory response, including chemokine receptor 3 (CCR3), chemokine ligand 21(CCL21), and chemokine ligand 13 (CCL13)[21]. Thirdly, Tseng-Rogenski et al reported that interleukin 6 may alter the localization of human MutS homolog 3 (hMSH3), and induce dMMR tumours [22], implying that chronic inflammation may lead to dMMR colorectal tumours.

The results of our study are in line with those obtained by Park and group (16), while they are inconsistent with those published by Pine and group, who report a lack of correlation between NLR and MMR status [12]. There might be several reasons underlying this discordance. The study published by Pine and group involved a small sample size, with 55 dMMR and 286 pMMR CRC patients. In addition, there was heterogeneity in the patient population, with reference to tumour stage. A heterogeneous patient population was analysed as a single group, which probably affected statistical significance parameters. Our results show that metastatic status of the CRC significantly influences the association between NLR and dMMR. This may help reconcile the discordance between results published by Park et al and Pine et al. We also found that NLR and CRP had prognostic value in pMMR CRC patients, in accordance with literature. However, neither NLR nor CRP was a predictive factor in dMMR CRC patients, suggesting that MMR status impacts the prognostic value of NLR and CRP.

In our study, we found that early stage dMMR CRC patients had higher systemic inflammatory responses than pMMR CRC patients. When distant metastasis was found, the systemic inflammatory responses were comparable between dMMR and pMMR CRC patients. One reason for this observation might be that dMMR CRC patients are more prone to display somatic mutations. As a result, dMMR CRC could be recognized by the immune system earlier, and induce systemic inflammatory response at an early stage. Previous studies have also reported that systemic inflammation is caused by the release of several pro-inflammatory cytokines, including interleukin (IL) IL-1, IL-3, and IL-6. Interleukins like IL-6 are known to alter the localization of MutS homolog 3 (hMSH3), leading to mismatch repair defects. The development of distant metastasis could lead to elevation of various cytokines and cause a universal systemic inflammatory response, regardless ofMMR status [23, 24].

The main limitation of this study is that it is a retrospective study from a single hospital. In addition, we did not analyse microsatellite instability status of patients. Another limitation is that since most study participants were diagnosed in recent years, OS of all patients could not be assessed.

In summary, this study suggests that patients with dMMR status may be associated with higher neutrophil counts and CRP levels in early stage CRC, and that NLR has prognostic value only in pMMR CRC. Further studies are needed to validate our results. 


\section{Supplementary Material}

Supplementary tables.

http://www.jcancer.org/v09p3093s1.pdf

\section{Acknowledgments}

This work was supported by grants from the Natural Science Foundation of Guangdong, China (2015A030313010), Science and Technology Program of Guangzhou, China (1563000305) and National Natural Science Foundation of China (81272641 and 81572409). We would like to thank Editage (www.editage.cn) for English language editing. The data in the study were deposited in the Research Data Deposit system of Sun Yat-sen University Cancer [RDDA2018000761].

\section{Competing Interests}

The authors have declared that no competing interest exists.

\section{References}

1. Le DT, Uram JN, Wang H, Bartlett BR, Kemberling H, Eyring AD, et al. PD-1 Blockade in Tumors with Mismatch-Repair Deficiency. N Engl J Med. 2015; 372: 2509-20.

2. Li SK, Martin A. Mismatch Repair and Colon Cancer: Mechanisms and Therapies Explored. Trends Mol Med. 2016; 22: 274-89.

3. Xiao Y, Freeman GJ. The microsatellite instable subset of colorectal cancer is a particularly good candidate for checkpoint blockade immunotherapy. Cancer Discov. 2015; 5: 16-8

4. Benatti P, Gafa R, Barana D, Marino M, Scarselli A, Pedroni M, et al. Microsatellite instability and colorectal cancer prognosis. Clin Cancer Res. 2005; 11: 8332-40.

5. Guastadisegni C, Colafranceschi M, Ottini L, Dogliotti E. Microsatellite instability as a marker of prognosis and response to therapy: a meta-analysis of colorectal cancer survival data. Eur J Cancer. 2010; 46: 2788-98.

6. Popat S, Hubner R, Houlston RS. Systematic review of microsatellite instability and colorectal cancer prognosis. J Clin Oncol. 2005; 23: 609-18.

7. Ward R, Meagher A, Tomlinson I, O'Connor T, Norrie M, Wu R, et al. Microsatellite instability and the clinicopathological features of sporadic colorectal cancer. Gut. 2001; 48: 821-9.

8. Li Y, Liang L, Dai W, Cai G, Xu Y, Li X, et al. Prognostic impact of programed cell death-1 (PD-1) and PD-ligand 1 (PD-L1) expression in cancer cells and tumor infiltrating lymphocytes in colorectal cancer. Mol Cancer. 2016; 15: 55.

9. Llosa NJ, Cruise M, Tam A, Wicks EC, Hechenbleikner EM, Taube JM, et al. The vigorous immune microenvironment of microsatellite instable colon cancer is balanced by multiple counter-inhibitory checkpoints. Cancer Discov. 2015; 5: 43-51.

10. He W, Yin C, Guo G, Jiang C, Wang F, Qiu H, et al. Initial neutrophil lymphocyte ratio is superior to platelet lymphocyte ratio as an adverse prognostic and predictive factor in metastatic colorectal cancer. Med Oncol. 2013; 30: 439.

11. Kantola T, Vayrynen JP, Klintrup K, Makela J, Karppinen SM, Pihlajaniemi T, et al. Serum endostatin levels are elevated in colorectal cancer and correlate with invasion and systemic inflammatory markers. Br J Cancer. 2014; 111: 1605-13.

12. Pine JK, Morris E, Hutchins GG, West NP, Jayne DG, Quirke P, et al. Systemic neutrophil-to-lymphocyte ratio in colorectal cancer: the relationship to patient survival, tumour biology and local lymphocytic response to tumour. $\mathrm{Br} \mathrm{J}$ Cancer. 2015; 113: 204-11.

13. Zhang XY, Zhang G, Jiang Y, Liu D, Li MZ, Zhong Q, et al. The prognostic value of serum $\mathrm{C}$-reactive protein-bound serum amyloid $\mathrm{A}$ in early-stage lung cancer. Chin J Cancer. 2015; 34: 335-49.

14. Park JH, Watt DG, Roxburgh CS, Horgan PG, McMillan DC. Colorectal Cancer, Systemic Inflammation, and Outcome: Staging the Tumor and Staging the Host. Ann Surg. 2016; 263: 326-36.

15. Dolan RD, McSorley ST, Park JH, Watt DG, Roxburgh CS, Horgan PG, et al. The prognostic value of systemic inflammation in patients undergoing surgery for colon cancer: comparison of composite ratios and cumulative scores. Br J Cancer. 2018.

16. Ishizuka M, Nagata H, Takagi K, Iwasaki $Y$, Shibuya N, Kubota K. Clinical Significance of the C-Reactive Protein to Albumin Ratio for Survival After Surgery for Colorectal Cancer. Ann Surg Oncol. 2016; 23: 900-7.
17. Park JH, Powell AG, Roxburgh CS, Horgan PG, McMillan DC, Edwards J. Mismatch repair status in patients with primary operable colorectal cancer: associations with the local and systemic tumour environment. Br J Cancer. 2016; 114: 562-70.

18. Klintrup K, Makinen JM, Kauppila S, Vare PO, Melkko J, Tuominen H, et al. Inflammation and prognosis in colorectal cancer. Eur J Cancer. 2005; 41: 2645-54.

19. Mesker WE, Junggeburt JM, Szuhai K, de Heer P, Morreau H, Tanke HJ, et al. The carcinoma-stromal ratio of colon carcinoma is an independent factor for survival compared to lymph node status and tumor stage. Cell Oncol. 2007; 29: 387-98.

20. Casbon AJ, Reynaud D, Park C, Khuc E, Gan DD, Schepers K, et al. Invasive breast cancer reprograms early myeloid differentiation in the bone marrow to generate immunosuppressive neutrophils. Proc Natl Acad Sci U S A. 2015; 112: E566-75.

21. Missiaglia E, Jacobs B, D'Ario G, Di Narzo AF, Soneson C, Budinska E, et al. Distal and proximal colon cancers differ in terms of molecular, pathological, and clinical features. Ann Oncol. 2014; 25: 1995-2001.

22. Tseng-Rogenski SS, Hamaya Y, Choi DY, Carethers JM. Interleukin 6 alters localization of hMSH3, leading to DNA mismatch repair defects in colorectal cancer cells. Gastroenterology. 2015; 148: 579-89.

23. Sharp SP, Avram D, Stain SC, Lee EC. Local and systemic Th17 immune response associated with advanced stage colon cancer. J Surg Res. 2017; 208: 180-6.

24. Wang W, Li X, Zheng D, Zhang D, Huang S, Zhang X, et al. Dynamic changes of peritoneal macrophages and subpopulations during ulcerative colitis to metastasis of colorectal carcinoma in a mouse model. Inflamm Res. 2013; 62: 669-80. 